\title{
NIL, NILPOTENT AND PI-ALGEBRAS
}

\author{
VLADIMÍR MÜLLER \\ Institute of Mathematics, Academy of Sciences of the Czech Republic \\ Žitná 25, 115 67 Praha 1, Czech Republic
}

The notions of nil, nilpotent or PI-rings (= rings satisfying a polynomial identity) play an important role in ring theory (see e.g. [8], [11], [20]). Banach algebras with these properties have been studied considerably less and the existing results are scattered in the literature. The only exception is the work of Krupnik [13], where the Gelfand theory of Banach PI-algebras is presented. However, even this work has not get so much attention as it deserves.

The present paper is an attempt to give a survey of results concerning Banach nil, nilpotent and PI-algebras.

The author would like to thank to J. Zemánek for essential completion of the bibliography.

I. Nil and nilpotent Banach algebras. All algebras are complex; we do not assume the existence of the unit element.

The set of all positive integers will be denoted by $\mathbb{N}$. For $n \in \mathbb{N}$ we denote by $\mathcal{P}_{n}$ the set of all complex polynomials in $n$ non-commuting variables.

Definition 1. Let $A$ be a Banach algebra. We say that

(1) $A$ is nil $\Leftrightarrow$ every element of $A$ is nilpotent, i.e. for every $x \in A$ there is $n \in \mathbb{N}$ such that $x^{n}=0$,

(2) $A$ is nilpotent $\Leftrightarrow$ there exists $m \in \mathbb{N}$ such that $x_{1} \ldots x_{m}=0$ for all $x_{1}, \ldots, x_{m} \in A$,

(3) $A$ is algebraic $\Leftrightarrow$ for every $x \in A$ there is a polynomial $p \in \mathcal{P}_{1}, p \neq 0$ such that $p(x)=0$,

(4) $A$ is locally finite $\Leftrightarrow$ every finite subset of $A$ generates a finite-dimensional subalgebra,

1991 Mathematics Subject Classification: 46H05, 46H20.

The paper is in final form and no version of it will be published elsewhere. 
(5) $A$ is a PI-algebra $\Leftrightarrow$ there exist $n \in \mathbb{N}$ and a polynomial $p \in \mathcal{P}_{n}, p \neq 0$, such that $p\left(x_{1}, \ldots, x_{n}\right)=0$ for all $x_{1}, \ldots, x_{n} \in A$,

(6) $A$ is locally $P I \Leftrightarrow$ for every sequence $\left\{x_{i}\right\}_{i=1}^{\infty} \subset A$ there exist $n \in \mathbb{N}$ and $p \in \mathcal{P}_{n}, p \neq 0$, such that $p\left(x_{1}, \ldots, x_{n}\right)=0$.

There are a number of relations among these notions:

Theorem 2 [15]. A Banach algebra is PI if and only if it is locally PI.

Theorem 3 ([1], cf. also [12], [5] and [10]). Let $A$ be a Banach algebra. The following properties are equivalent:

(a) A is algebraic,

(b) $A$ is locally finite,

(c) $\operatorname{Rad} A$ is nilpotent and codim $\operatorname{Rad} A<\infty$.

Theorem 4 [7]. A Banach algebra is nil if and only if it is nilpotent.

Thus we have

$$
(1) \Leftrightarrow(2) \Rightarrow(3) \Leftrightarrow(4) \Rightarrow(5) \Leftrightarrow(6) .
$$

The algebra of all $n \times n$ matrices is an example of an algebraic Banach algebra which is not nilpotent.

Any infinite-dimensional commutative semisimple Banach algebra is an example of a PI-algebra which is not algebraic (by Theorem 3).

The proof of Theorem 4 consists of two steps:

1. If every element of a Banach algebra $A$ is nilpotent then there exists $n \in \mathbb{N}$ such that $x^{n}=0$ for every $x \in A$. This (as well as many other results in this paper) is a consequence of the Baire category theorem.

2. If $x^{n}=0$ for every $x \in A$ then there exists $m \in \mathbb{N}$ such that $x_{1} \ldots x_{m}=0$ for all $x_{1}, \ldots, x_{m} \in A$. This is the so-called Nagata-Higman theorem (see [16], [9] or [11], Appendix C), which is purely algebraic (actually, it has recently been discovered that this theorem was already proved by Dubnov and Ivanov [4]).

Denote by $d(n)$ the least integer such that $x_{1} \ldots x_{d(n)}=0$ for all $x_{1}, \ldots, x_{d(n)}$ $\in A$ provided that $x^{n}=0(x \in A)$. An interesting open problem is to determine the exact value of $d(n)$. The best known bounds are ([14], [18])

$$
\frac{n(n+1)}{2} \leq d(n) \leq n^{2} .
$$

For a survey of results about the Nagata-Higman theorem, see [6].

II. Approximate properties. A natural approximate analogue to the notion of a nilpotent element $x$ of a Banach algebra $A$ is the concept of quasinilpotent elements:

$$
x \in A \text { is quasinilpotent } \Leftrightarrow r(x)=\lim _{n \rightarrow \infty}\left\|x^{n}\right\|^{1 / n}=0 .
$$


There are several possibilities to define approximative versions of nil or nilpotent algebras (cf. [2]).

Definition 5. Let $A$ be a Banach algebra. Then

(1) $A$ is radical (= topologically nil) $\Leftrightarrow$ every element of $A$ is quasinilpotent, i.e.

for every $x \in A$,

$$
\lim _{n \rightarrow \infty}\left\|x^{n}\right\|^{1 / n}=0
$$

(2) $A$ is uniformly topologically nil $\Leftrightarrow \lim _{n \rightarrow \infty} S_{A}(n)=0$ where

$$
S_{A}(n)=\sup \left\{\left\|x^{n}\right\|^{1 / n}: x \in A,\|x\|=1\right\},
$$

(3) $A$ is topologically nilpotent $\Leftrightarrow \lim _{n \rightarrow \infty}\left\|x_{1} \ldots x_{n}\right\|^{1 / n}=0$ for every sequence $x_{1}, x_{2}, \ldots \in A$ with $\left\|x_{i}\right\|=1(i=1,2, \ldots)$,

(4) $A$ is uniformly topologically nilpotent $\Leftrightarrow \lim _{n \rightarrow \infty} N_{A}(n)=0$ where

$$
N_{A}(n)=\sup \left\{\left\|x_{1} \ldots x_{n}\right\|^{1 / n}: x_{1}, \ldots, x_{n} \in A,\left\|x_{i}\right\|=1(i=1, \ldots, n)\right\} .
$$

These notions are related in the following manner:

THEOREM 6 [3]. A Banach algebra is topologically nilpotent if and only if it is uniformly topologically nilpotent.

Theorem 7 [2]. If $A$ is a topologically nilpotent Banach algebra then $A$ is uniformly topologically nil. If $A$ is commutative then these two notions are equivalent.

Thus we have

$$
(4) \Leftrightarrow(3) \Rightarrow(2) \Rightarrow(1)
$$

and for commutative Banach algebras

$$
(4) \Leftrightarrow(3) \Leftrightarrow(2) \Rightarrow(1) \text {. }
$$

An example of a uniformly topologically nil algebra which is not topologically nilpotent can be found in [3] (clearly such an example is non-commutative).

If $A$ is a uniformly topologically nil then $A$ is clearly radical and it is not difficult to find an example of an (even commutative) radical Banach algebra which is not uniformly topologically nil: consider the $\ell^{1}$-algebra $A$ generated by elements $x_{1}, x_{2}, \ldots$ which satisfy $\left\|x_{i}\right\|=1, x_{i} x_{j}=0 \quad(i \neq j)$, and $\left\|x_{i}^{i}\right\|=1$, $x_{i}^{i+1}=0$.

Another interesting notion is the following (see [19], [22]; for a related concept see also [17]):

For a bounded subset $M$ of a Banach algebra $A$ define $\|M\|=\sup \{\|x\|: x$ $\in M\}$ and $r(M)=\lim _{n \rightarrow \infty}\left\|M^{n}\right\|^{1 / n}$ (the spectral radius of $M$ ), where

$$
M^{n}=\left\{x_{1} \ldots x_{n}: x_{i} \in M, i=1, \ldots, n\right\} .
$$


Note that in this notation $N_{A}(n)=\left\|B_{A}^{n}\right\|^{1 / n}$, where $B_{A}$ is the closed unit ball of $A$, and $A$ is topologically nilpotent if and only if $r\left(B_{A}\right)=0$. An interesting open problem (see [22]) is the following

Problem $\left(^{1}\right)$. Let $A$ be a radical Banach algebra. Is then $A$ finitely quasinilpotent, i.e. $r(F)=0$ for every finite subset $F \subset A$ ?

It is easy to see that this is true for commutative Banach algebras. A positive answer is known also if the algebra $A$ consists of compact operators in a Banach space (see [21]).

The example mentioned above is an example of a commutative finitely quasinilpotent algebra which is not uniformly topologically nil.

A countable version of this problem has a negative answer. As an example, take the set $M=\left\{x_{1}, x_{2}, \ldots\right\}$ in the $\ell^{1}$-algebra with generators $x_{1}, x_{2}, \ldots$ which satisfy the relation $x_{i} x_{j}=0$ for $j \neq i+1$. In other words, the elements of the algebra are of the form

$$
y=\sum_{i<j} \alpha_{i, j} x_{i} x_{i+1} \ldots x_{j-1}
$$

where the complex coefficients $\alpha_{i, j}$ satisfy $\|y\|=\sum_{i<j}\left|\alpha_{i, j}\right|<\infty$.

III. Gelfand theory of Banach PI-algebras. In this section we give a survey of the Gelfand theory for Banach PI-algebras (see [13]).

To avoid technical difficulties we formulate the results only for algebras with unit.

If $A$ is a Banach PI-algebra without unit, then its unification is also a PIalgebra (by Theorem 2), so that the results can be modified for algebras without unit in the obvious way.

Among all polynomial identities, the standard identities play an important role. Define

$$
e_{n}\left(x_{1}, \ldots, x_{n}\right)=\sum_{\sigma \in S_{n}} \operatorname{sign}(\sigma) x_{\sigma(1)} \ldots x_{\sigma(n)},
$$

where $S_{n}$ is the set of all permutations of $\{1, \ldots, n\}$ and $\operatorname{sign}(\sigma)= \pm 1$ for $\sigma$ even (odd). Clearly $e_{2}\left(x_{1}, x_{2}\right)=x_{1} x_{2}-x_{2} x_{1}$.

ExAmples (of Banach PI-algebras). 1. Any commutative Banach algebra $A$ satisfies the standard identity $e_{2}(x, y)=0$ for all $x, y \in A$.

2. Let $A$ be a finite-dimensional Banach algebra, $\operatorname{dim} A=n$. Then it is easy to show that

$$
e_{n+1}\left(x_{1}, \ldots, x_{n+1}\right)=0 \quad\left(x_{1}, \ldots, x_{n+1} \in A\right) .
$$

3. An important particular case is the algebra $M_{n}$ of all $n \times n$ (complex) matrices. By 2 , the algebra $M_{n}$ satisfies the polynomial identity $e_{n^{2}+1}\left(x_{1}, \ldots, x_{n^{2}+1}\right)$ $=0$. Actually, a better result is true:

$\left.{ }^{1}\right)$ Editorial note: See also Question 15 in the article of V. S. Shul'man in this volume. 
TheOREm 8 (Amitzur, Levitzky). The algebra $M_{n}$ satisfies the standard identity

$$
e_{2 n}\left(x_{1}, \ldots, x_{2 n}\right)=0 \quad\left(x_{1}, \ldots, x_{2 n} \in M_{n}\right) .
$$

It can be shown that $M_{n}$ satisfies no polynomial identity of degree $\leq 2 n$.

Denote by $\mathcal{F}_{n}$ the class of all unital Banach algebras $A$ which satisfy the standard identity $e_{n}\left(x_{1}, \ldots, x_{n}\right)=0$ for all $x_{1}, \ldots, x_{n} \in A$.

Clearly $\mathcal{F}_{2}$ is the class of all commutative unital Banach algebras so that the classes $\mathcal{F}_{n}$ can be considered as a generalization of commutative Banach algebras. Further, $M_{n} \in \mathcal{F}_{2 n}$ by Theorem 8.

Denote by $\mathcal{M}(A)$ the set of all maximal two-sided ideals in a Banach algebra $A$. For $M \in \mathcal{M}(A)$ denote by $\pi_{A}$ the canonical homomorphism $\pi_{M}: A \rightarrow A / M$.

It is well-known that if $A$ is a commutative Banach algebra, then maximal ideals are of codimension $1, \bigcap\{M: M \in \mathcal{M}(A)\}=\operatorname{Rad} A$, and $x \in A$ is invertible $\Leftrightarrow \pi_{M}(x) \neq 0(M \in \mathcal{M}(A))$.

The following theorem is a generalization of these facts:

Theorem 9 ([13], Theorem 21.1). Let $A$ be a Banach algebra of class $\mathcal{F}_{2 n}$. Then

(a) For every $M \in \mathcal{M}(A)$ the algebra $A / M$ is isomorphic to $M_{l}$ for some $l \leq n$,

(b) $\bigcap\{M: M \in \mathcal{M}(A)\}=\operatorname{Rad} A$,

(c) $x \in A$ is invertible $\Leftrightarrow \pi_{M}(x)$ is invertible for every $M \in \mathcal{M}(A)$.

If $A$ is a Banach PI-algebra then it can be shown that $A$ satisfies a homogeneous multilinear identity, i.e. there exist $n \in \mathbb{N}$ and complex coefficients $\alpha_{\sigma}$ $\left(\sigma \in S_{n}\right)$, not all zero such that

$$
\sum_{\sigma \in S_{n}} \alpha_{\sigma} x_{\sigma(1)} \ldots x_{\sigma(n)}=0 \quad\left(x_{1}, \ldots, x_{n} \in A\right) .
$$

However, not every Banach PI-algebra satisfies a standard identity. Consider the exterior algebra $A$ (with unit) generated by elements $e_{1}, e_{2}, \ldots$ with multiplication $e_{i} e_{j}=-e_{j} e_{i}(i, j \in \mathbb{N})$. This algebra is PI as $(x y-y x) z-z(x y-y x)=0$ $(x, y, z \in A)$ and it can be easily seen that $A$ does not satisfy any standard identity.

The situation changes when we consider semisimple Banach algebras or, equivalently, Banach PI-algebras modulo radical.

TheOrem 10. Let A be a unital Banach algebra. The following statements are equivalent:

(1) $A / \operatorname{Rad} A$ is a PI-algebra.

(2) $A / \operatorname{Rad} A \in \mathcal{F}_{2 n}$ for some $n \in \mathbb{N}$.

(3) There exists $m \in \mathbb{N}$ such that $x \in A$ is invertible $\Leftrightarrow \pi(x)$ is invertible for all representations (i.e. unit-preserving homomorphisms) $\pi: A \rightarrow M_{m}$. 
(4) There exists $m \in \mathbb{N}$ such that $x \in A$ is invertible $\Leftrightarrow \pi(x)$ is invertible for all representations $\pi: A \rightarrow M_{m}$ with the property

$$
\left|(\pi(y))_{i, j}\right| \leq\|y\| \quad(y \in A, i, j=1, \ldots, m) .
$$

Proof. The equivalence $(1) \Leftrightarrow(2) \Leftrightarrow(x \in A$ is invertible $\Leftrightarrow \pi(x)$ is invertible for all representations $\left.\pi: A \rightarrow M_{l}(l \leq n)\right)$ can be found in [13], Theorems 22.1 and 22.2 .

To show the equivalence with (3), take $m=n$ ! (in fact $m=n^{2}$ is sufficient), so that for any representation $\pi: A \rightarrow M_{l}$ with $l \leq n$ there is a representation $\tilde{\pi}: A \rightarrow M_{m}, \tilde{\pi}=\bigoplus_{i=1}^{m / l} \pi$.

For the equivalence with (4), see [13], Lemma 25.1.

For commutative Banach algebras there is a 1-1 correspondence between maximal ideals and multiplicative functionals. For PI-algebras the role of multiplicative functionals is played by finite-dimensional representations, but a finitedimensional representation is not uniquely determined by its kernel. Property (4) of the previous theorem shows that it is sufficient to consider a compact set of representations.

Let $A$ be a unital Banach algebra such that $A / \operatorname{Rad} A$ is a PI-algebra. Let $m \in \mathbb{N}$ be the integer from the previous theorem. Denote by $\mathcal{K}$ the set of all representations $\pi: A \rightarrow M_{m}$ with the property

$$
\left|(\pi(y))_{i, j}\right| \leq\|y\| \quad(y \in A, i, j=1, \ldots, m) .
$$

We define a topology on $\mathcal{K}$ in the following way: the basis of neighborhoods of an element $\pi \in \mathcal{K}$ is formed by the sets

$$
U_{x_{1}, \ldots, x_{k}, \varepsilon}=\left\{\pi^{\prime} \in \mathcal{K}:\left\|\pi^{\prime}\left(x_{i}\right)-\pi\left(x_{i}\right)\right\|<\varepsilon, i=1, \ldots, k\right\}
$$

where $x_{1}, \ldots, x_{k} \in A, \varepsilon>0$.

Clearly $\mathcal{K}$ is a compact set. Denote by $C\left(\mathcal{K}, M_{m}\right)$ the algebra of all continuous functions from $\mathcal{K}$ to $M_{m}$ and define a mapping $G: A \rightarrow C\left(\mathcal{K}, M_{m}\right)$ by

$$
G(a)(\pi)=\pi(a) \quad(a \in A, \pi \in \mathcal{K}) .
$$

THEOREM 11. Let $A$ be a unital Banach algebra such that $A / \operatorname{Rad} A$ is a PI-algebra. Then the mapping $G: A \rightarrow C\left(\mathcal{K}, M_{m}\right)$ has the following properties:

(1) $G$ is a continuous homomorphism,

(2) $a \in A$ is invertible $\Leftrightarrow G(a)$ is invertible in $C\left(\mathcal{K}, M_{m}\right)$,

(3) $G(a)=0 \Leftrightarrow a \in \operatorname{Rad} A$,

(4) $\sigma(a)=\bigcup_{\pi \in \mathcal{K}} \sigma(G(a)(\pi))$ so that $r(a)=\max _{\pi \in \mathcal{K}} r(G(a)(\pi))$.

\section{References}

[1] P. G. Dixon, Locally finite Banach algebras, J. London Math. Soc. 8 (1974), 325-328.

[2] -, Topologically nilpotent Banach algebras and factorization, Proc. Roy. Soc. Edinburgh Sect. A 119 (1991), 329-341. 
[3] P. G. Dixon and V. Müller, A note on topologically nilpotent Banach algebras, Studia Math. 102 (1992), 269-275.

[4] J. Dubnov et V. Ivanov, Sur l'abaissement du degré des polynômes en affineurs, C. R. (Doklady) Acad. Sci. URSS 41 (1943), 95-98.

[5] J. Duncan and A. W. Tullo, Finite dimensionality, nilpotents and quasinilpotents in Banach algebras, Proc. Edinburgh Math. Soc. 19 (1974/75), 45-49.

[6] E. Formanek, The Nagata-Higman Theorem, Acta Appl. Math. 21 (1990), 185-192.

[7] S. Grabiner, The nilpotency of Banach nil algebras, Proc. Amer. Math. Soc. 21 (1969), 510.

[8] I. N. Herstein, Noncommutative Rings, Carus Math. Monographs 15, Math. Assoc. Amer., Wiley, 1968.

[9] G. Higman, On a conjecture of Nagata, Proc. Cambridge Philos. Soc. 52 (1956), 1-4.

[10] R. A. Hirschfeld and B. E. Johnson, Spectral characterization of finite-dimensional algebras, Indag. Math. 34 (1972), 19-23.

[11] N. Jacobson, Structure of Rings, third edition, Amer. Math. Soc. Colloq. Publ. 37, Amer. Math. Soc., Providence, R.I., 1968.

[12] I. Kaplansky, Ring isomorphisms of Banach algebras, Canad. J. Math. 6 (1954), $374-381$.

[13] N. Ya. Krupnik, Banach Algebras with Symbol and Singular Integral Operators, Birkhäuser, Basel, 1987.

[14] E. N. Kuzmin, On the Nagata-Higman Theorem, in: Mathematical Structures - Computational Mathematics-Mathematical Modeling, Proceedings dedicated to the sixtieth birthday of Academician L. Iliev, Sofia, 1975, 101-107 (in Russian).

[15] V. Müller, Kaplansky's theorem and Banach PI-algebras, Pacific J. Math. 141 (1990), 355-361.

[16] M. Nagata, On the nilpotency of nil-algebras, J. Math. Soc. Japan 4 (1952), 296-301.

[17] K. M. Przyłuski and S. Rolewicz, On stability of linear time varying infinite-dimensional discrete-time systems, Systems Control Lett. 4 (1984), 307-315.

[18] Y. P. Razmyslov, Trace identities of full matrix algebras over a field of characteristic zero, Izv. Akad. Nauk SSSR Ser. Mat. 38 (1974), 723-756 (in Russian).

[19] G. C. Rota and W. G. Strang, A note on the joint spectral radius, Indag. Math. 22 (1960), 379-381.

[20] L. H. Rowen, Polynomial Identities in Ring Theory, Academic Press, New York, 1980.

[21] V. S. Shul'man, On invariant subspaces of Volterra operators, Funct. Anal. Appl. 18 (1984), 85-86.

[22] Yu. V. Turovskiur, Spectral properties of certain Lie subalgebras and the spectral radius of subsets of a Banach algebra, in: Spectral Theory of Operators and its Applications, No. 6, Elm, Baku, 1985, 144-181 (in Russian). 